\title{
Langerhans cell histiocytosis of the temporal bone in a child.
}

A Chakravarti, Rajeev Gupta, J K Sahni

Department of Otorhinolaryngology Head \& Neck Surgery,

Lady Hardinge Medical College, New Delhi

\section{Abstract:}

Langerhans cell histiocytosis of temporal bone is a benign tumor like condition with variable clinical course. This rare clinical entity may mimic common ENT clinical condition like otitis media, mastoiditis and otitis externa. A high index of suspicion is required to reach to a diagnosis of langerhans cell histiocytosis to prevent delay in proper management of this disease.

Key words: langerhans cell histiocytosis, temporal bone.

\section{Introduction}

Langerhans' cell histiocytosis (LCH) refers to a group of diseases whose primary pathogenesis is an abnormal polyclonal proliferation of Langerhans cells. [1] Langerhans cells, which are macrophages that are normally present only in the dermis, are the characteristics of this disease. [2] This disease is divided in three subtypes: eosinophilic granuloma (EG), Hand-Schuller-Christian disease (HSC), and Letterer-Siwe (LS) disease. [3] The incidence of LCH is approximately 5.4 per million. It is a rare disease with a male predominance. It mainly affects children of age between 1 to 4 years. $[1,4]$ The clinical presentation of LCH depends upon the site of involvement. It can range from a multifocal to a solitary lesion. It usually affects the head and neck, mainly the skull base, in $60 \%$ of cases. [3,4] The temporal bone is involved in $19-25 \%$ of cases and the involvement is bilateral in a third of all cases. [2,5] The petrous ridge of the temporal bone is the most commonly affected area. [5] This disease has an unpredictable natural history varying from a rapidly, fatal progressive disease to spontaneous resolution. [1]

We report a case of Langerhans' cell histiocytosis of temporal bone who was initially misdiagnosed and treated for chronic otitis media with mastoiditis. The diagnosis and management of this rare clinical entity is being discussed.

\section{Case Report}

A two year old child presented to ENT outpatient department with history of right ear discharge with swelling over right post auricular region and intermittent fever with no chills and rigor for last 2 months. Child was diagnosed as chronic otitis media with mastoiditis and received 10 days course of intravenous antibiotics. In view of no improvement, patient was referred to our centre for further management. Clinical examination revealed tender right post auricle swelling of size $3 \times 3 \mathrm{~cm}$ with soft tissue bulge visible in external auditory canal obscuring visualization of tympanic membrane. There was no facial nerve palsy, sign of sepsis or intracranial involvement. General physical examination revealed seborrheic rash over right side of temporal, parietal region of scalp and back of the patient (Fig 1). Contrast enhanced computed tomography (CECT) scans showed a significant destructive bone lesion with enhancing soft tissue component of size approximately $5 \times 4.6 \times 3 \mathrm{~cm}$ involving petrous, squamous and mastoid part of right temporal bone( sparing apical petrous, inner and middle ear) causing obliteration of external auditory canal with displacement and compression of underlying brain but no evidence of intraaxial extension (Fig 2). Patient underwent biopsy from postauricle region under general anaesthesia with differential diagnosis of histiocytosis, rhabdomyosarcoma or lymphoid malignancy. Disease was found to be infiltrating temporalis muscle with bony dehiscence. Histopathology analysis revealed an sheets of polygonal cells with abundant eosinophilic cytoplasm, lobulated indented nuclei 
showing grooving prominent admixture of eosinophils with few histiocytic giant cells suggestive of Langerhans cell histiocytosis(Fig 3). Positive immunohistochemical staining for S-100 protein confirmed the diagnosis. Complete blood count reveal raised ESR $(84 \mathrm{~mm} / \mathrm{hr})$. Liver function test, coagulation studies and urine osmolarity test were normal. Skeletal survey and bone marrow examination ruled out multi organ involvement.

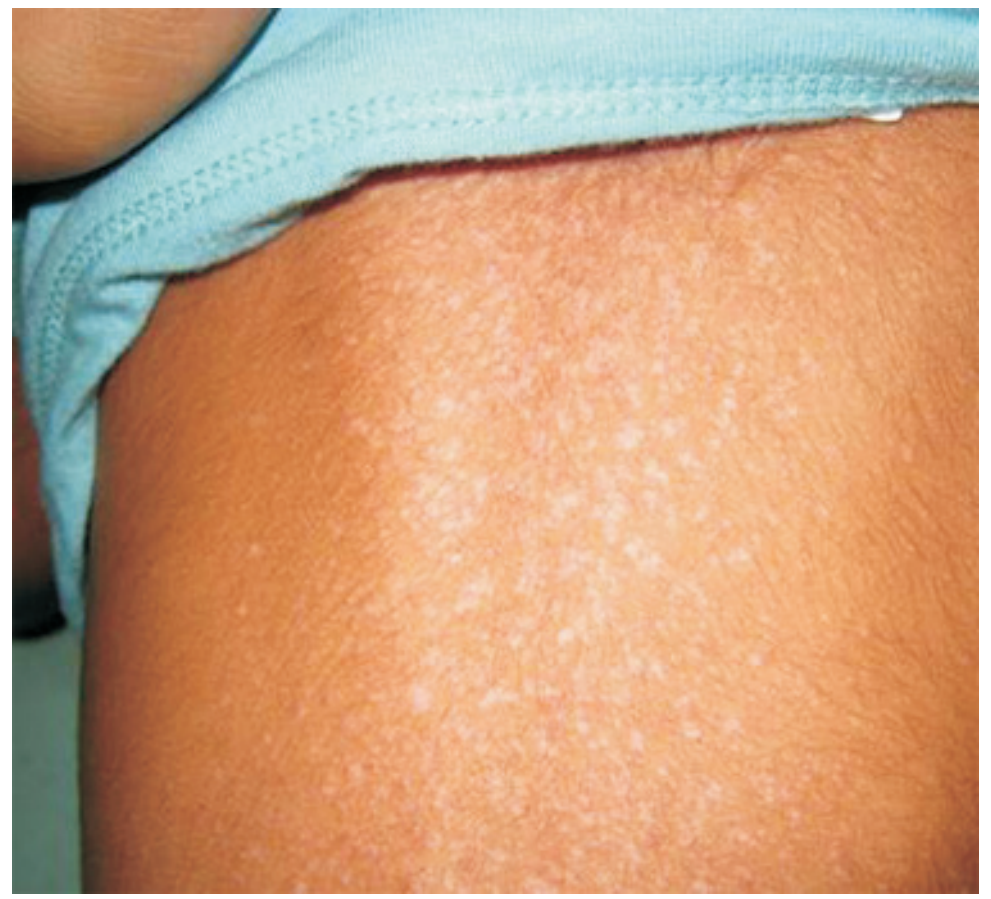

Figure 1: Seborrheic Rash over back of patient

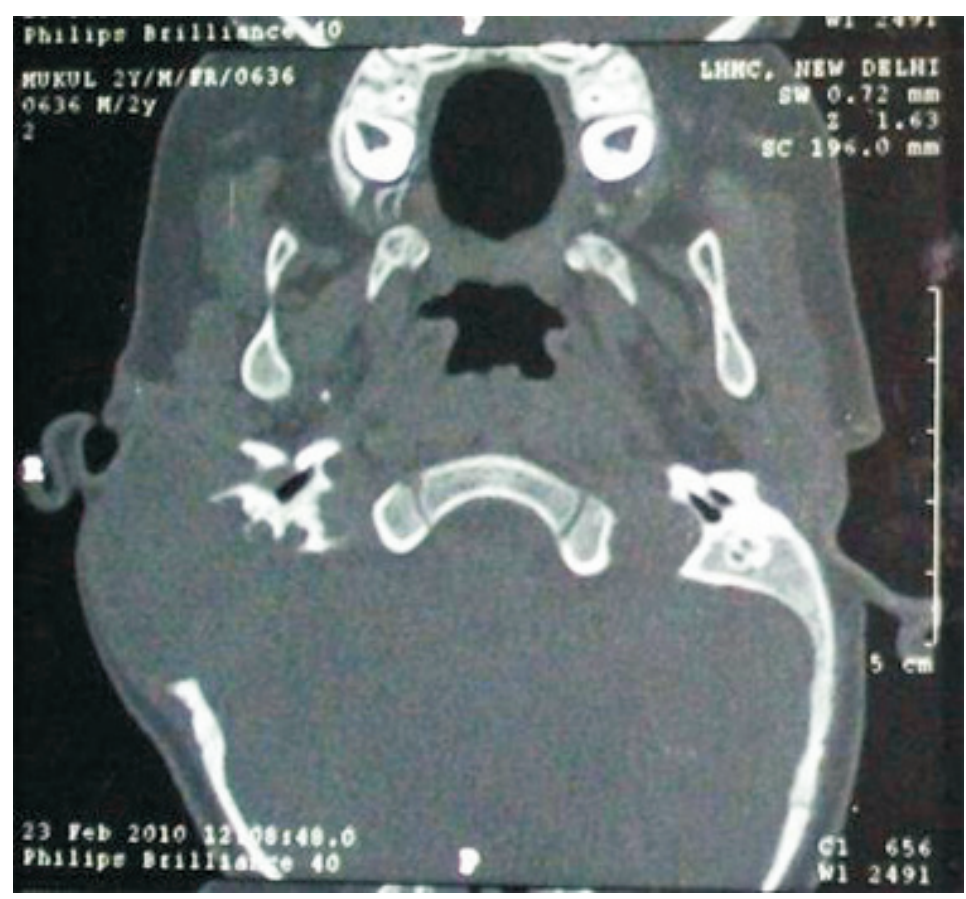

Figure 2: CECT Temporal Bone (Axial Cut) showing destructive bony lesion with enhancing soft tissue component of size involving petrous, squamous and mastoid part of right temporal bone. 


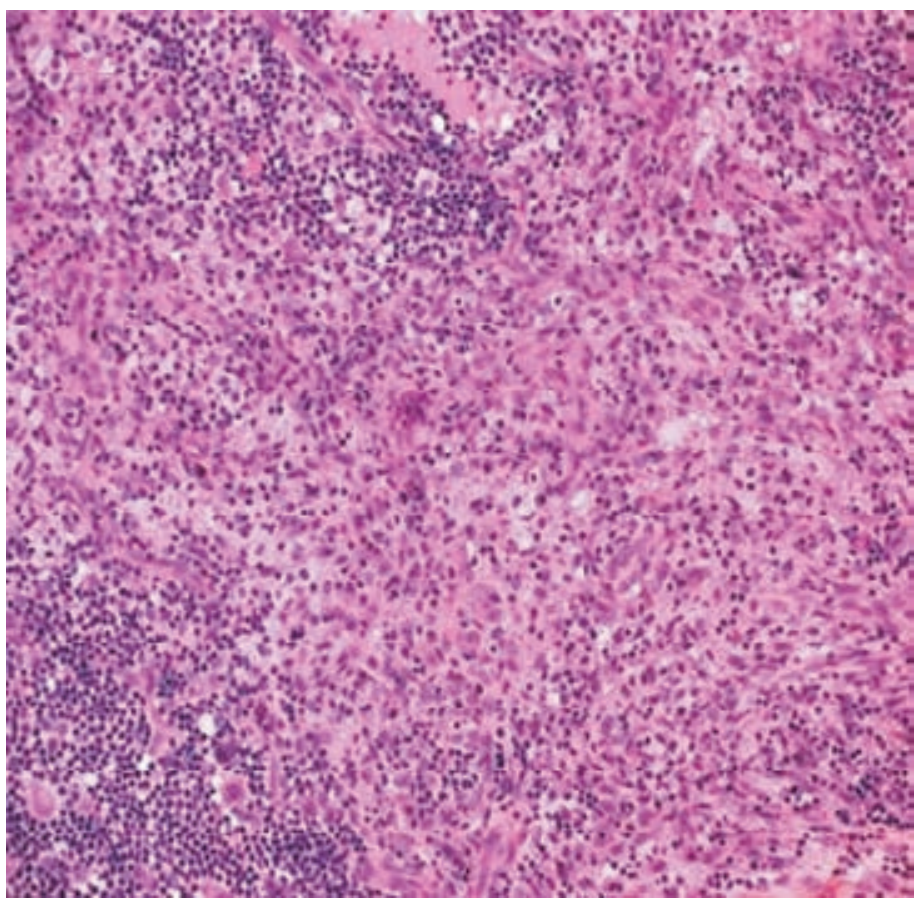

Figure 3: Microphotograph (H\&E stain with magnification 200x) showing polygonal cell with abundant cytoplasm with histiocytotic cell suggestive of Langerhans Cell Histiocytosis.

Patient was treated with intralesional triamcinolone $(40 \mathrm{mg})$ injection given in temporal mass three times with space of 1 week and oral prednisolone $(1 \mathrm{mg} / \mathrm{kg} /$ day) tapered over period of 6 weeks. Beclomethasone skin ointment was used for skin lesion. After 6 weeks of therapy post auricle swelling reduced considerably and skin lesion disappeared. The patient is in complete remission with no evidence of recurrence with 1 year of follow up.

\section{Discussion}

Lichtenstein and Jaffe coined the term eosinophilic granuloma in 1940.[1] In 1953, Lichtenstein linked this disease with Hand-Schuller-Christian disease and Letterer-Siwe disease, and proposed to combine these three entities under the name 'histiocytosis X', on the basis of their similar histopathological findings.[7] 'Langerhans cell histiocytosis' was officially adopted in 1986 during the Workshop on Childhood Histiocytoses. [8]

It is still debated whether the proliferation of Langerhans cells is of neoplastic or reactive (e.g. due to viral infection) origin. Many classifications have been proposed over the years. With regard to treatment and prognosis, patients are best categorized as having either restrictive or extensive disease. [9] Restrictive disease consists of monostotic and polyostotic eosinophilic granulomas of the bone or skin. Extensive forms include Hand-Schuller-Christian disease and Letterer-Siwe disease. Currently, different staging systems are used. Classification of the writing group of the Histiocytes Society defines three stages of $\mathrm{LCH}$ : (stage 1) single system disease; (stage 2) multisystem disease; (stage 3) multisystem disease with evidence of organ dysfunction. [10] According to this staging present case patient was categorized in stage 2 after ruling out multi organ dysfunction.

Patients of histiocytosis of temporal bone most commonly present with purulent aural discharge, post-auricular swelling, vertigo, hearing loss and otalgia. Facial palsy is a rare finding (less than 5 per cent). An assessment of cranial nerves, orbits and cerebellar function should be performed in all cases. This presentation of histiocytosis can be confused with acute otitis media or mastoiditis which may delay the treatment as happened in the present case.

Head and neck lesions should first be investigated radiologically. Bone lesions typically show a 'punched-out' appearance, with no reactive sclerosis, although a local periosteal reaction can occur. In the mastoid, CT scanning reveals a coalescence of cells with extension to the surrounding structures (bony canal walls, tegmentum and posterior fossa) which can appear similar to cholesteatoma. Macroscopically, the lesion is often reddish or brown. Gold standard investigation for histiocytosis is characteristic picture on histopathology with positive immunohistochemical staining for S-100 protein and CD 1 antigen. 
The treatment of patients with Langerhans cell histiocytosis varies widely according to the extent of the disease. Isolated bony lesions can often be observed, unless there is pain or deformity, because of a high rate of spontaneous remission. Single system disease can be treated with local injectable intralesional steroids [10]. In single system bone disease, injection of methylprednisolone into the bone can be extremely useful and may lead to the resolution of the lesion. We have found intralesional steroid to be highly effective in our case with no recurrence on 1 year follow up. Extensive disease requires a systemic approach comprising chemotherapy often combined with corticosteroids. The principal agents used are vinblastine and etoposide. 1, 2-CdA can be administered in refractory cases. Factors indicating a poor prognosis include an age of less than two years, multiple organ involvement and organ failure (i.e. liver, lungs and haematopoietic system). Prognosis of temporal bone involvement in histiocytosis is good having limited organ involvement, although some patients, even if responsive to medical therapies, may progress to disseminated disease. Patient should be kept under regular follow even after remission.

A high index of suspicion from otolaryngologist is required to recognize otological manifestation of Langerhans' cell histiocytosis. Otological manifestation of histiocytosis can mimic more common disease like otitis externa, aural polyp, acute mastoiditis and chronic ottitis media which delay diagnosis and treatment. A early diagnosis and multidisciplinary approach is required for proper staging of disease to plan best management of case as treatment varies from case to case.

\section{References}

1. Boston $M$, Craig SD. Langerhans cell histiocytosis of the temporal bone and skull base. Am. J. Otolaryngol. 2002; 23 (4): 246-248.

2. Martini A, Aimoni C, Trevisani M, Marangoni P. Langerhans cell histiocytosis: report of a case with temporal localization, Int. J. Pediatr. Otorhinolaryngol. 2000; 55:51-56.

3. Bayazit $Y$, Sirikci A, Bayaram M, Kanlikama M, Demir A, Bakir K, Eosinophilic granuloma of the temporal bone. Auris Nasus Larynx 2001 ; 28: 99-102.

4. de Brito Macedo Ferreira LM, de Carvalho JD, Pereira ST, Tavares MG. Histiocytosis X of the temporal bone. Rev. Bras. Otorrinolaringol. (Engl. Ed.). 2006; 72: 575.

5. Azouz EM, Saigal G, Rodriguez MM, Podda A. Langerhans' cell histiocytosis: pathology, imaging and treatment of skeletal involvement. Pediatr. Radiol. 2005; 35:103-1 15.

6. Lichtenstein L, Jaffe HL. Eosinophilic granuloma of bone - with report of a case. Am J Pathol 1940; 16:595-604.

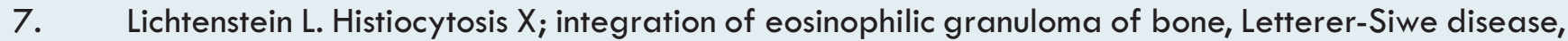
and Schuller- Christian disease as related manifestations of a single nosologic entity. AMA Arch Pathol 1953; 56: 84-102.

8. Chu A, Favara BE, Ladisch S, Nezelof C, Prichard J. Report and recommendations of the workshop on the childhood histiocytoses: concepts and controversies. Med Pediatr Oncol 1986;1 14: 1 16-1 17.

9. Egeler RM, D'Angio GJ. Langerhans cell histiocytosis. J Pediatr 1995; 1 27: 1-11.

10. Campos MK, Viana MB, de Oliveira BM, Ribeiro DD, Silva CM. Langerhans cell histiocytosis: a 16year experience, J. Pediatr. (Rio J.) 2007; 83: 79-86. 